\title{
Differential neural activation to friends and strangers links interdependence to empathy
}

\author{
Meghan L. Meyer • Carrie L. Masten • Yina Ma • \\ Chenbo Wang • Zhenhao Shi • Naomi I. Eisenberger • \\ Matthew D. Lieberman • Shihui Han
}

Accepted: 16 November 2014

(C) Springer-Verlag Berlin Heidelberg 2014

\begin{abstract}
Two competing views implicate interdependence in empathy. One suggests that interdependence may generally enhance empathy (Woltin et al., British Journal of Social Psychology 50:553-562, 2011), whereas another suggests that interdependence enhances empathy for targets with whom one is in a relationship, at the cost of decreasing empathy for strangers (Markus and Kitayama, Perspectives on Psychological Science 5(4):420-430, 2010). Here, we show evidence in support of the latter account. We observed that trait-level interdependence positively correlated with trait-level empathic abilities in perspective-taking and empathic concern. However, using lan empathy for social exclusion paradigm, we found that neural responses to a friend's compared to a stranger's social exclusion (vs. inclusion) differentially related to interdependence, perspective-taking and empathic concern. During the observation of a friend's social exclusion (vs. inclusion), neural responses in the medial prefrontal cortex (MPFC), and to a lesser
\end{abstract}

M. L. Meyer $(\bowtie) \cdot$ C. L. Masten · N. I. Eisenberger · M. D. Lieberman

UCLA Psychology Department, 1285 Franz Hall, Box 951563, Los Angeles, CA 90095-1563, USA e-mail:mlmeyer@ucla.edu

Y. Ma

Lieber Institute for Brain Development, Baltimore, USA

C. Wang $\cdot$ S. Han

PKU-IDG/McGovern Institute for Brain Research, Peking University, Beijing, People's Republic of China

\section{Z. Shi}

The Annenberg Public Policy Center, University of Pennsylvania, 202 S. 36th Street, Philadelphia, PA 19104-3806, USA

\section{S. Han $(\varangle)$}

Department of Psychology, Peking University, 5 Yiheyuan Road, Beijing 100871,

People's Republic of China

e-mail: shan@pku.edu.cn 
extent the dorsal anterior cingulate cortex (dACC) and anterior insula (AI), positively correlated with self-reported trait interdependence, perspective-taking, and empathic concern. In contrast, during the observation of a stranger's social exclusion (vs. inclusion), neural responses in the MPFC, and to a lesser extent the dACC and AI, negatively correlated with self-reported trait interdependence, perspectivetaking and empathic concern. These findings suggest that while trait interdependence may correspond with enhanced ability to empathize, as indicated by selfreport measures, interdependent individuals may preferentially recruit this ability for close others relative to strangers.

Keywords Empathy $\cdot$ Interdependence $\cdot$ MPFC $\cdot$ fMRI

\section{Introduction}

A major tenet within social psychology is that cultural influences on how people conceptualize the self should have consequences for their cognition, affect, and behavior. A predominant view is that an interdependent self (or, perceiving oneself and one's experiences as connected to, or interdependent with, others), leads to various pro-social responses, including enhanced empathy (Woltin et al. 2011). In support of this view, it has been shown that interdependence is associated with accurate judgment of targets' embarrassment during social evaluation (Woltin et al. 2011), forgoing self-interested decisions for others' benefit (Gardner et al. 2004), and heightened perspective-taking skills (Wu and Keysar 2007). Together, these findings point to the pro-social consequences of interdependence, implicating it as a trait that may enhance empathic processes.

An alternative view suggests interdependence may not lead to a general increase in empathy. Instead, interdependence may increase ingroup/outgroup distinction by simultaneously incorporating individuals with whom one has a relationship with into the self ("we") and sharpening the boundary between the interdependent self and outgroup members ("them"; Iyengar et al. 1999; Markus and Kitayama 2010; Triandis 1972, 1989). As a result, any person with whom the interdependent self does not have a relationship (e.g., strangers) may be vulnerable to outgroup biases. Evidence in support of this alternative comes from research on intergroup processes, which finds that in addition to enhancing pro-social responses towards ingroup members, interdependence also increases negative biases towards minimal outgroup members (e.g., physically similar strangers; Leung 1988; Leung and Bond 1984; Ma-Kellams and Blascovich 2012; Wong and Hong 2005). For example, Wong and Hong (2005) found that priming interdependence relative to a neutral prime in Chinese-American participants enhanced cooperation with friends but decreased cooperation with strangers. Similarly, priming interdependence in Chinese-native participants prior to their viewing strangers' pain led to reduced electrophysiological responses associated with pain processing (Jiang et al. 2013), suggesting they experienced less vicarious pain than is typically observed during empathy for pain paradigms (e.g., Singer et al. 2004). Interestingly, empathic processes are also 
susceptible to outgroup biases: empathic behavioral and neural processes are moderated by extreme ingroup/outgroup distinctions, such as racial (Avenanti et al. 2010; Xu et al. 2009; Sheng and Han 2012; Sheng et al. 2013) and rival (Hein et al. 2010) status. Thus, if interdependence widens the net of who is susceptible to outgroup biases (e.g., not just extreme outgroup members, such as rivals, but also minimal outgroup members, such as physically similar strangers), reduced empathy toward strangers may be one such instance of increased outgroup bias.

Distinct bodies of research on the neural basis of interdependence on the one hand, and empathy on the other hand, point to patterns of brain activation that may clarify how trait interdependence affects empathy. One brain region in particularmedial prefrontal cortex (MPFC)/Brodmann Area 10 (BA10)—seems to engage during three relevant processes. First, MPFC is known to engage during selfprocessing (Denny et al. 2012; Kelley et al. 2002) and also supports interdependent self-representations (Chiao et al. 2009, 2010; Zhu et al. 2007; Ma et al. 2014; Ng et al. 2010; Wang et al. 2013). That is, thinking about the self and thinking about a close other (separately) shows overlapping neural activation in MPFC in Chinese, but not Western, subjects (Zhu et al. 2007). Similarly, priming interdependence activates this region when reflecting on personality traits of a close other ( $\mathrm{Ng}$ et al. 2010), as does thinking about the self in relation to close others (Chiao et al. 2009, 2010), suggesting that interdependent representations of the self are coded in MPFC. Second, MPFC has been associated with empathy (Lamm et al. 2007; Rameson et al. 2012). For example, MPFC has been linked to empathy for a target's anxiety (Morelli et al. 2012) and social and emotional suffering (Bruneau et al. 2012b; Masten et al. 2011), and even appears to track with participants' selfreported empathy (Rameson et al. 2012). In fact, MPFC is particularly associated with the cognitive components of empathy, such as perspective-taking (D'Argembeau et al. 2007; Jackson et al. 2006; Lamm et al. 2007) and empathic concern (Danziger et al. 2009), which require the representation of another person's mental experience. Thus, representing another's mind via MPFC may be more likely when that person is already embedded in self-representations within MPFC. Third, MPFC decreases when participants are asked to consider the plight of distant, or extreme outgroup members (Bruneau et al. 2012a; Harris and Fiske 2006, 2007), perhaps reflecting a neural signature of reduced empathy towards outgroup members.

Taken together, these neuroimaging findings offer response patterns to look for to test the competing predictions regarding how interdependence relates to empathy. If interdependence generally enhances empathy through MPFC activity, then individuals with stronger trait interdependence should show greater MPFC activation during empathy for both close others (friends) and non-close others (strangers). In contrast, if interdependence enhances empathy for close others (friends), but reduces empathy for non-close others (strangers), then activation in MPFC should differentially correlate with strength of interdependence, increasing activity in response to close others in empathy eliciting situations, but decreasing in response to strangers in empathy eliciting situations.

To test these competing hypotheses, participants underwent functional magnetic resonance imaging (fMRI) while they observed a friend's and stranger's exclusion from the Cyberball game (Eisenberger et al. 2003), and outside of the scanner 
completed questionnaire measures of trait empathy and interdependence. Building on the finding that interdependence corresponds with accurate judgments for targets' emotional responses to social evaluation (Woltin et al. 2011), we expected that construing oneself as more interdependent would also result in greater neural activity in MPFC to social exclusion (for either friends and strangers, or differentially for friends vs. strangers). In addition to MPFC, two other brain regions - dorsal anterior cingulate cortex (dACC) and anterior insula (AI) - are associated with empathy, particularly the affective (rather than cognitive) components of empathy, such as vicarious emotions (Singer et al. 2004; Zaki and Ochsner 2012). Thus, although the dACC and AI have not been directly linked to trait interdependence, we also examined whether these regions' responses tied interdependence to trait empathy, and if so, whether they showed a positive association for friends and strangers, or a differential association for friends versus strangers.

\section{Methods}

\section{Participants}

Sixteen Chinese university students (12 females/4 males), $M$ age $=21.69$, $\mathrm{SD}=2.12$, participated in the study. All participants were right-handed, had normal or corrected-to-normal vision, and reported no neurological or psychiatric history. Participants completed written consent in accordance with the Peking University Ethics Committee and were paid for participation. Other results from participants in this study have been reported (Meyer et al. 2013), however all results reported in the present manuscript are orthogonal (i.e., statistically independent) to those previously reported.

\section{Procedure}

Participants came to the scanner with a gender-matched close friend. Participants were introduced to the "Cyberball game" in which people play a live, computerized balltossing game with other players over the Internet. Each participant underwent fMRI scanning while they observed what they believed was their friend's Cyberball game and what they believed was a stranger's Cyberball game. In reality, these Cyberball games were prerecorded videos that were the same for all participants, and not actually their friend or a stranger playing live. In addition to instructions indicating which target's game they were to watch prior to the actual viewing, during the game a photograph of their friend in a neutral facial expression (taken by the experimenter before the scan) and a photograph of a gender-matched stranger in a neutral facial expression were presented at the bottom of the screen for each corresponding game (see Meyer et al. 2013 for a pictorial display of this design). Each Cyberball game was one scanning run and lasted 2 min $45 \mathrm{~s}$. Each game began with an inclusion period (24 s) in which all players received the ball an equal number of times. After the inclusion period, one player (either the participant's friend or a stranger, depending on the run) was excluded from the game. The order of the friend and stranger Cyberball 
games was counterbalanced across participants to ensure that observed neural differences for friend's and stranger's exclusion (vs. inclusion) was not an artifact of the order in which these games were observed. The firsthand experience of Cyberball exclusion reliably evokes the experience of social exclusion and feelings of distress (Eisenberger and Lieberman 2004; Eisenberger et al. 2003) and similar feelings during empathy for Cyberball exclusion (Masten et al. 2011; Meyer et al. 2013).

Following the scan session, participants completed the individualism and collectivism attitude scale (Triandis and Gelfand 1998) designed to measure traitlevel interdependence (i.e., how much individuals' self-views are connected to others in the social environment) and independence (i.e., how much individuals' self-views are discrete from social relationships) as well as the interpersonal reactivity index (IRI; Davis 1983), which measures four components of empathy: perspective-taking (the tendency to adopt the point of view of others), empathic concern (the tendency to feel sympathy or compassion for the suffering of others), personal distress (the tendency to experience others' suffering as aversive), and fantasy (the tendency to experience fictional characters as though their experience is one's own).

fMRI data acquisition

fMRI data were collected with a Siemens Trio 3-Tesla head-only MRI scanner at the Chinese Academy of Sciences Institute of Biophysics. Participants observed Cyberball games via an LCD screen in the scanner. Whole-brain blood oxygenation-level-dependent functional scans were acquired during the Cyberball task (echoplanar T2-weighted gradient-echo, $\mathrm{TR}=2,000 \mathrm{~ms}, \mathrm{TE}=30 \mathrm{~ms}$, flip angle $=90^{\circ}$, matrix size $=64 \times 64 \times 32$ axial slices, FOV $=24 \times 24 \mathrm{~cm} ; 4 \mathrm{~mm}$ thick, voxel size $=3.44 \times 3.44 \times 5 \mathrm{~mm})$. Additionally, a set of high-resolution T1-weighted structural images were acquired coplanar with the functional scans (matrix size $256 \times 256 \times 76$ matrix with a spatial resolution of $1 \times 1 \times 1 \mathrm{~mm}, \mathrm{TR}=2,600 \mathrm{~ms}$, $\mathrm{TE}=3.02 \mathrm{~ms}$, inversion time $=900 \mathrm{~ms}$, flip angle $=8^{\circ}$, thickness $=1 \mathrm{~mm}$ ) .

\section{Data analysis}

Neuroimaging data were preprocessed and analyzed with SPM5 (the Wellcome Trust Centre for Neuroimaging, London, UK). Preprocessing for each participant's images included skull-stripping using brain extraction tool (Smith 2002) to enhance preprocessing accuracy, spatial realignment to correct for head motion, normalization into a standard stereotactic space as defined by the Montreal Neurological Institute, and spatial smoothing using an $8 \mathrm{~mm}$ Gaussian kernel, full width at half maximum, to increase the signal-to-noise ratio. Brain imaging data was modeled as a block design, with an inclusion period and exclusion period (each $24 \mathrm{~s}$ ), as well as 'null periods' that were modeled to account for variance in neural activation associated with the remaining $1 \mathrm{~min} 48 \mathrm{~s}$. Prior to the onset of the inclusion block there were $9 \mathrm{~s}$ of fixation, which served as the implicit baseline.

To test whether interdependence was associated with empathic neural responses to the friend and stranger's social exclusion, we conducted region of interest (ROI) analyses for the MPFC, dACC and AI. Because MPFC and dACC are large 
anatomical structures, we created $10 \mathrm{~mm}$ spheres around peaks reported in publications measuring neural responses to psychological constructs similar to those in our study in interdependent Chinese samples. For the MPFC ROI, we made a sphere around a peak of MPFC (BA10) reported by Zhu et al. (2007) shown to be associated with overlapping representations between the self and close others among Chinese participants. Selecting the coordinate from Zhu et al. (2007) seemed particularly appropriate given our interest in testing a region sensitive to interdependent representations between self and close others, because this peak was shown to engage when Chinese participants thought about themselves and when they thought about a close other, whereas Western participants only activated this region when thinking about the self. For the dACC ROI, because we wanted to measure neural responses known to be sensitive to empathy within an interdependent Chinese sample, we made a sphere around a peak of dACC reported by Xu et al. (2009) to be associated with empathy in Chinese subjects. Because the anatomy is more constrained and well defined for AI, we used structural ROIs for AI constructed in PickAtlas (Maldjian et al. 2003), using templates from the atlas of Tzourio-Mazoyer et al. (2002). ROI analyses computed, for each subject, the average activation in the voxels within each ROI separately during the observation of the friend's exclusion (vs. inclusion) and during the observation of the stranger's exclusion (vs. inclusion). Next, we correlated participants' MPFC, dACC and AI parameter estimates comparing exclusion versus inclusion from the ROI analyses with scores on the trait measures of interdependence and empathy. Consistent with fMRI whole-brain analyses, ROI analyses were one-tailed. To compare within subject correlations between (1) ROI activation in response to a friend's exclusion with trait measures and (2) ROI activation in response to a stranger's exclusion with trait measures, we computed a $t$-statistic that takes into account the degree to which the two tests are correlated, and therefore uses $N-3$ (rather than $N-2$ ) degrees of freedom (Williams 1959; Steiger 1980). We followed up our ROI analyses with whole-brain regression analyses. These whole-brain regressions were thresholded at $p<.005,149$ voxel extent, which corresponds with a false-discovery rate of $5 \%$ across the whole brain, as estimated by a Monte Carlo simulation implemented in AlphaSim in AFNI (Cox 1996).

\section{Results}

\section{Questionnaires}

Prior to performing correlation analyses, we assessed whether our questionnaire measures were accurately measuring our constructs of interest. To do so, we computed Cronbach's $\alpha$ s as an index of questionnaire reliability. The interdependence score was highly reliable $(\alpha=.84)$, however the independence score was far less reliable $(\alpha=.42)$, and this value was not able to be increased by the exclusion of any questions on the sub-scale. This is not entirely surprising, as given the known cultural differences in interdependence and independence (Markus and Kitayama 1991), the independence questions were likely not answered similarly among our 
strongly interdependent sample. Given the low reliability, correlational analyses with the independence score are not reported in the "Results" section. ${ }^{1}$ For each empathy sub-scale, appropriate $\alpha$ values were met (perspective-taking $\alpha=.72$, empathic concern $\alpha=.70$, personal distress $\alpha=.68$, and fantasy scale $\alpha=.78$ ) by removing one to two items from each sub-scale. Trait interdependence/ independence scores can fall on a continuum from 1 to 98 and Empathy IRI subscale scores can range from 1 to 5 . Our sample had the following scores on these measures: mean interdependence $=79.19(\mathrm{SD}=9.09$, range 62-92), mean independence $=62.88 \quad(\mathrm{SD}=6.02$, range 54-76), mean perspective-taking $=3.4$ $(\mathrm{SD}=.71$, range 2.2-4.4), mean empathic concern $=3.66 \quad(\mathrm{SD}=.59$, range $=2-4.5)$, mean personal distress $=3.03(\mathrm{SD}=.67$, range $=2-4)$, mean fantasy $=3.73(\mathrm{SD}=.77$, range $2.5-5)$.

\section{Behavioral correlations}

\section{Questionnaire correlations}

Consistent with our predictions, there was a positive correlation between interdependence scores and the perspective-taking $(r=.66, p=.006)$ and empathic concern $(r=.60, p=.02)$ empathy sub-scales (Fig. 1). In contrast, interdependence scores did not correlate with personal distress $(r=.-07, p=.80)$ or fantasy ( $r=.16, p=.57$ ) empathy sub-scales.

\section{Brain-behavior correlations}

We next examined whether neural responses to a friend's exclusion (vs. inclusion) and stranger's exclusion (vs. inclusion) differentially related to trait empathy and interdependence. Correlation comparisons for the friend versus the stranger conditions revealed significant differences in the correlation between MPFC and perspective-taking $[t(13)=3.44, \quad p=.002]$, MPFC and empathic concern $[t(13)=2.95, \quad p=.006], \quad$ and $\mathrm{MPFC}$ and interdependence $[t(13)=2.18$, $p=.02] .^{2}$ Post-hoc analyses showed that MPFC during the friend condition positively correlated with individual difference measures [perspective-taking $(r=.60, p=.007)$, empathic concern $(r=.52, p=.02)$, and interdependence $(r=.53, p=.02)]$, whereas MPFC during the stranger condition negatively correlated with individual difference measures [perspective-taking $(r=-.49$, $p=.03)$, empathic concern $(r=-.48, p=.03)$ and (a marginal trend) with

\footnotetext{
${ }^{1}$ Correlations between trait independence and neural activation to the friend's exclusion (vs. inclusion) were non-significant $(p$ 's $>.21)$. The correlation between trait independence and neural activation to the stranger's exclusion (vs. inclusion) were non-significant in the dACC and AI ROIs ( $p$ 's $>.10$ ), however activation in the MPFC ROI during the stranger's exclusion (vs. inclusion) was negatively correlated $(r=-.71, p<.005)$ with independence. Given the low reliability of the independence questionnaire in this sample, however, this result should be interpreted with caution.

2 Note that the degrees of freedom is 13 because a third degree of freedom is lost due to comparing within subject correlations (Williams 1959; Steiger 1980).
} 


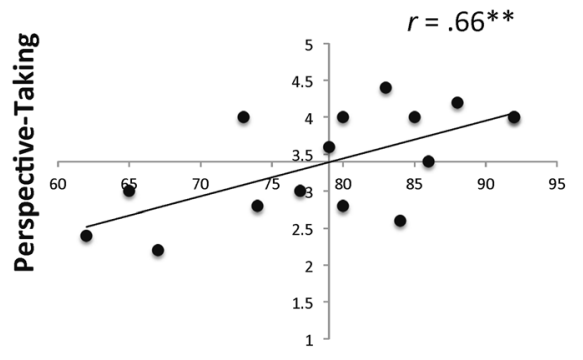

Interdependence

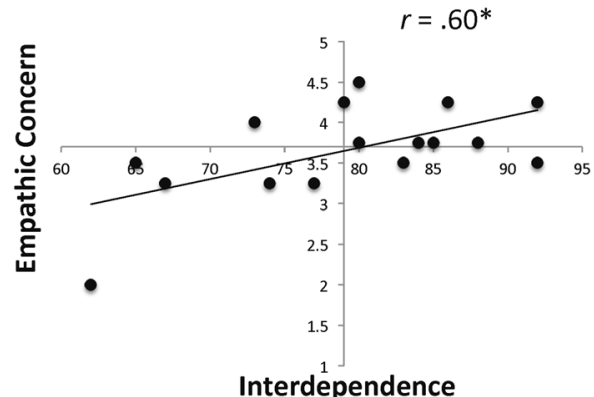

Interdependence

Fig. 1 Interdependence correlates with perspective-taking and empathic concern subscales of the Empathy IRI

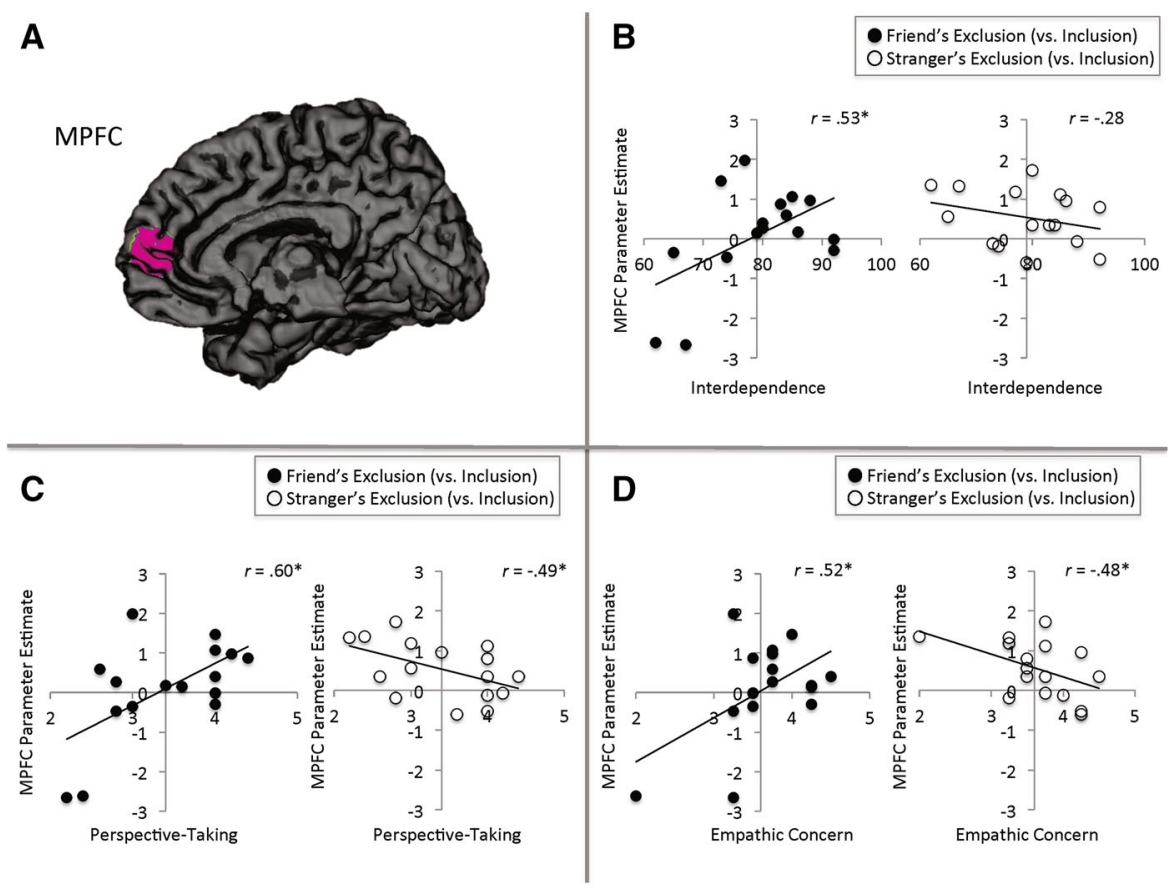

Fig. 2 a MPFC ROI. b The association between interdependence and MPFC activity during exclusion (vs. inclusion) to a friend and a stranger separately. c The association between perspective-taking and MPFC activity during exclusion (vs. inclusion) to a friend and a stranger separately. $\mathbf{d}$ The association between empathic concern and MPFC activity during exclusion (vs. inclusion) to a friend and a stranger separately. All of the pictured correlations for friends are significantly different than those shown for strangers

interdependence $(r=-.28, p=.14)]$. See Fig. 2 as well as Table 1 for a complete list of correlations.

Similar, though marginally significant, correlation comparisons between the friend condition and perspective-taking scores versus stranger condition and 
Table 1 Correlations between average activation in response to a friend's exclusion (vs. inclusion) and stranger's exclusion (vs. inclusion) in MPFC, dACC, and AI regions-of-interest and questionnaire measures

\begin{tabular}{|c|c|c|c|c|}
\hline & \multicolumn{2}{|c|}{ Friend's exclusion (vs. inclusion) } & \multicolumn{2}{|c|}{ Stranger's exclusion (vs. inclusion) } \\
\hline & $r$ & $p$ & $r$ & $p$ \\
\hline \multicolumn{5}{|l|}{ MPFC } \\
\hline Interdependence & 0.53 & 0.02 & -0.28 & 0.14 \\
\hline Perspective-taking & 0.60 & 0.007 & -0.49 & 0.03 \\
\hline Empathic concern & 0.52 & 0.02 & -0.48 & 0.03 \\
\hline Personal distress & 0.004 & 0.5 & 0.29 & 0.16 \\
\hline Fantasy scale & 0.39 & 0.07 & -0.28 & 0.14 \\
\hline \multicolumn{5}{|l|}{ dACC } \\
\hline Interdependence & 0.38 & 0.07 & -0.07 & 0.4 \\
\hline Perspective-taking & 0.34 & 0.09 & -0.19 & 0.24 \\
\hline Empathic concern & 0.02 & 0.47 & -0.25 & 0.18 \\
\hline Personal distress & 0.26 & 0.17 & -0.02 & 0.47 \\
\hline Fantasy scale & 0.06 & 0.41 & 0.04 & 0.45 \\
\hline \multicolumn{5}{|l|}{ Left anterior insula } \\
\hline Interdependence & 0.34 & 0.1 & 0.15 & 0.30 \\
\hline Perspective-taking & 0.35 & 0.09 & -0.32 & 0.11 \\
\hline Empathic concern & 0.11 & 0.34 & -0.14 & 0.31 \\
\hline Personal distress & 0.26 & 0.17 & 0.07 & 0.4 \\
\hline Fantasy scale & 0.02 & 0.47 & -0.06 & 0.41 \\
\hline \multicolumn{5}{|l|}{ Right anterior insula } \\
\hline Interdependence & 0.22 & 0.21 & 0.17 & 0.26 \\
\hline Perspective-taking & 0.13 & 0.31 & 0.002 & 0.5 \\
\hline Empathic concern & 0.16 & 0.28 & -0.08 & 0.39 \\
\hline Personal distress & 0.39 & 0.07 & -0.15 & 0.28 \\
\hline Fantasy scale & -0.02 & 0.48 & 0.03 & 0.45 \\
\hline
\end{tabular}

perspective-taking scores emerged for the dACC $[t(13)=1.28, p=.11]$ and AI $[t(13)=1.57, p=.07]$ ROIs (see Fig. 3). Interdependence also showed a marginal trend of differential correlation with AACC in the friend versus stranger condition $[t(13)=1.28, p=.15]$. See Table 1 for a complete list of these correlations.

\section{Whole-brain regression analyses}

In addition to the ROI results, we ran whole-brain regression analyses to see if, across all voxels in the brain, the MPFC shows a differential relationship with interdependence, perspective-taking, and empathic concern as a function of the target of exclusion. Consistent with the ROI results, these whole brain regressions showed that clusters of MPFC increase in response to observing the friend's exclusion (vs. inclusion) as a function of trait interdependence, perspective-taking, 


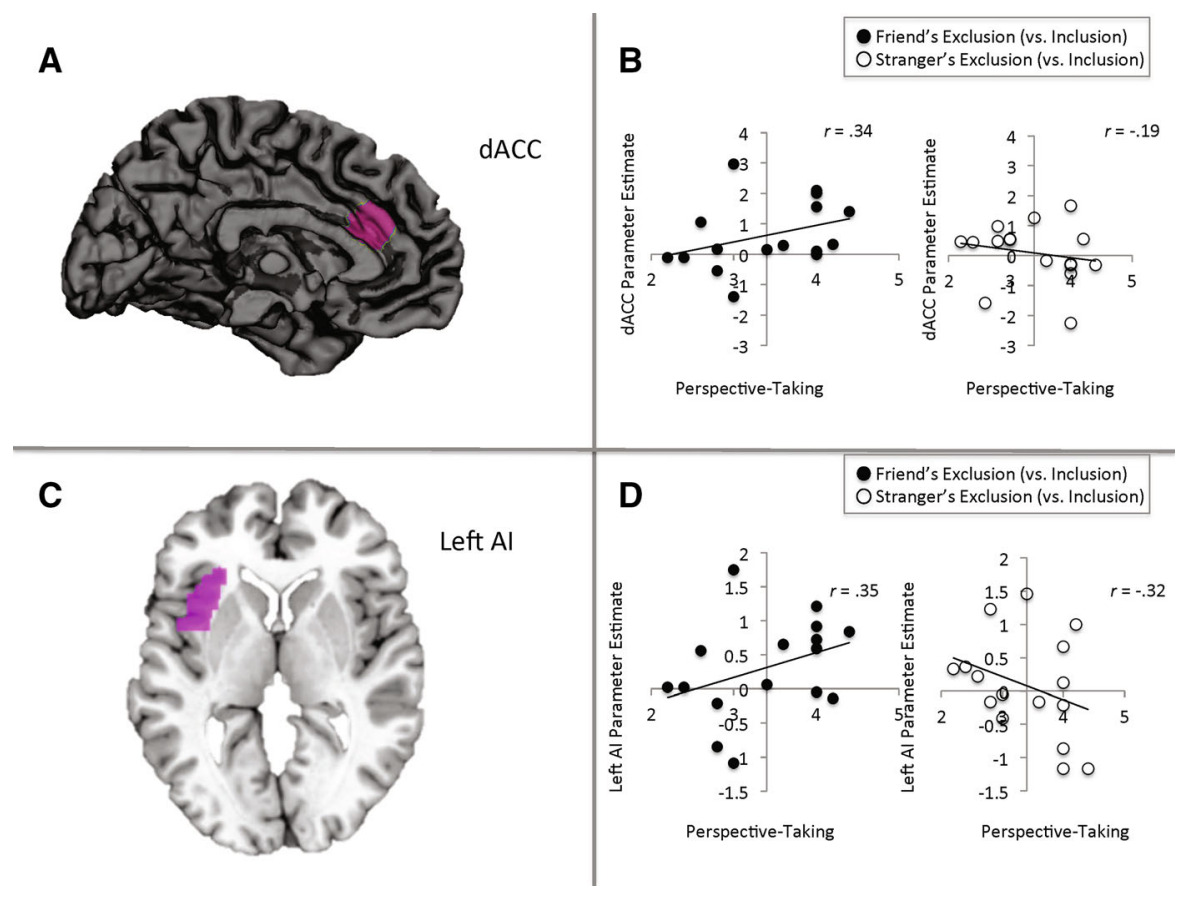

Fig. 3 a dACC ROI. b dACC activity in response to a friend's social exclusion (vs. inclusion) positively correlates with perspective-taking, whereas dACC activity in response to a stranger's social exclusion (vs. inclusion) negatively correlates with perspective-taking. c Left AI ROI. d Left AI activity in response to a friend's social exclusion (vs. inclusion) positively correlates with perspective-taking, whereas left AI activity in response to a stranger's social exclusion (vs. inclusion) negatively correlates with perspectivetaking

and empathic concern (Fig. 4; Table 2), but decrease in response to observing the stranger's exclusion (vs. inclusion) as a function of trait interdependence, and perspective-taking, though not empathic concern (Fig. 4; Table 3). Instead, empathic concern was associated with less activation in middle temporal gyrus.

\section{Discussion}

At the self-report level of analysis, we observed a correlation between interdependence and trait empathy. However, probing more deeply at neural responses during an empathy paradigm for both friends and strangers revealed support for the view that interdependence is associated with enhanced empathy for close others but decreased empathy for strangers. We found an empathy-by-target bias in MPFC, and to a lesser extent in AACC and AI, such that interdependence and trait empathy predicted increased activation in these regions while viewing a friend's exclusion but decreased activation in these regions while viewing a stranger's exclusion. These findings suggest that while interdependence may relate to empathic ability, 


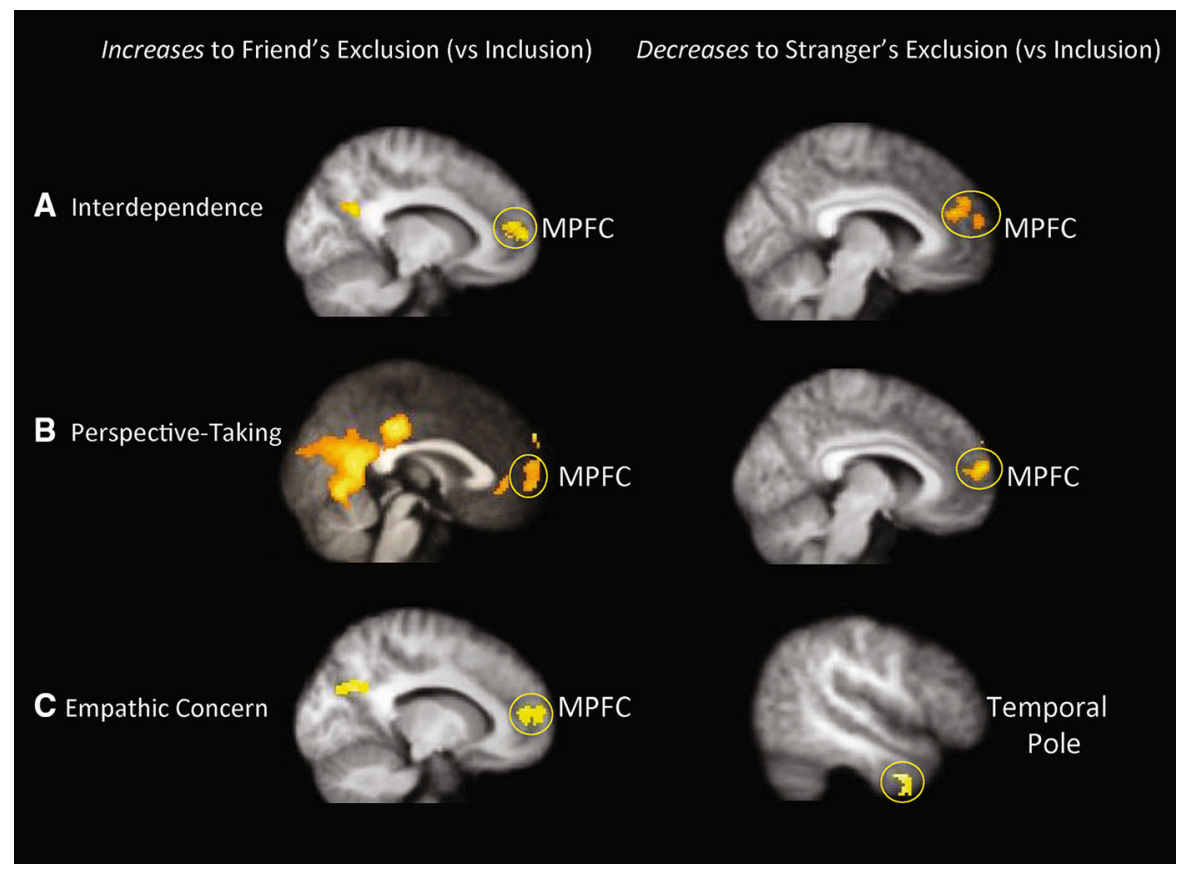

Fig. 4 Whole-brain regression results. Brain regions showing increases in activation to friend's exclusion (vs. inclusion; left panel) and decreases during the stranger's exclusion (vs. inclusion; right panel) as a function of $\mathbf{a}$ interdependence, $\mathbf{b}$ perspective-taking and $\mathbf{c}$ empathic concern

the neural mechanisms linking these constructs are sensitive to the relationship between a perceiver and a target of empathy.

These results help reconcile competing findings surrounding whether interdependence leads to a general increase in empathy, or a specific increase and decrease in empathy for close others and strangers. For example, empathic accuracy and perspective-taking are crucial elements of empathy (Davis 1983; Zaki et al. 2009a, b) and both have been shown to be enhanced towards a stranger as a function of interdependence (Woltin et al. 2011; Wu and Keysar 2007). However, these studies measured subjects' abilities in these two domains. In the study of empathic accuracy, participants were instructed to correctly identify a target's emotion (Woltin et al. 2011). In the study of perspective-taking, participants were instructed to correctly identify which object another person is thinking about based on which objects they could and could not see (Wu and Keysar 2007). However, these studies did not assess participants' motivation or tendency to empathize with the target independent of these instructions. Thus, interdependence may be generally associated with social cognitive abilities that have the potential to facilitate empathy across targets, but spontaneous, self-motivated empathy may be differentially reserved for those with whom one is interdependent relative to strangers. This reasoning may also help explain why perspective-taking and empathic concern scores from the Empathy IRI positively correlated with MPFC activation in 
M. L. Meyer et al.

Table 2 Whole-brain regression results

\begin{tabular}{|c|c|c|c|c|c|}
\hline \multicolumn{6}{|c|}{ Increases during the friend's exclusion (vs. inclusion) } \\
\hline Region & $x$ & $y$ & $z$ & $k$ & $t$ \\
\hline \multicolumn{6}{|l|}{ (A) Interdependence } \\
\hline \multirow[t]{3}{*}{ Posterior insula } & -40 & 0 & 12 & 180 & 5.57 \\
\hline & -50 & -8 & 6 & & 4.49 \\
\hline & -48 & -2 & 12 & & 4.34 \\
\hline \multirow[t]{2}{*}{ Precuneus } & 18 & -52 & 30 & 209 & 4.98 \\
\hline & 14 & -46 & 26 & & 4.09 \\
\hline \multirow[t]{2}{*}{ MPFC } & -16 & 54 & 14 & 165 & 4.66 \\
\hline & -12 & 64 & 6 & & 4.45 \\
\hline \multirow[t]{3}{*}{ Precuneus } & -14 & -58 & 26 & 348 & 3.94 \\
\hline & -6 & -54 & 26 & & 3.54 \\
\hline & -10 & -64 & 30 & & 3.47 \\
\hline \multicolumn{6}{|l|}{ (B) Perspective-taking } \\
\hline \multirow[t]{3}{*}{ Precuneus } & -14 & -54 & 22 & 4,805 & 6.86 \\
\hline & -6 & -30 & 36 & & 6.52 \\
\hline & -4 & -56 & 16 & & 6.16 \\
\hline \multirow[t]{3}{*}{ MPFC } & -12 & 62 & 4 & 1,109 & 6.76 \\
\hline & -18 & 54 & 12 & & 5.2 \\
\hline & -2 & 62 & 34 & & 4.98 \\
\hline \multirow[t]{3}{*}{ Superior frontal gyrus } & -30 & 48 & 36 & 168 & 6.02 \\
\hline & -26 & 56 & 34 & & 4.48 \\
\hline & -26 & 40 & 42 & & 3.35 \\
\hline \multirow[t]{3}{*}{ Postcentral gyrus } & -60 & -18 & 14 & 1,188 & 5.93 \\
\hline & -40 & 2 & 12 & & 5.92 \\
\hline & -48 & -2 & 14 & & 5.76 \\
\hline \multirow[t]{3}{*}{ Superior temporal gyrus } & 40 & -32 & 8 & 314 & 5.3 \\
\hline & 40 & -30 & -2 & & 4.84 \\
\hline & 38 & -22 & 0 & & 3.31 \\
\hline \multirow[t]{3}{*}{ Supplementary motor area } & 12 & -4 & 52 & 152 & 4.98 \\
\hline & 8 & 0 & 36 & & 3.58 \\
\hline & 8 & -8 & 34 & & 3.1 \\
\hline \multirow[t]{3}{*}{ Ventral lateral prefrontal cortex } & -48 & 52 & 6 & 229 & 4.86 \\
\hline & -50 & 42 & 6 & & 4.61 \\
\hline & -40 & 40 & -2 & & 4.31 \\
\hline \multirow[t]{2}{*}{ Postcentral gyrus } & 58 & -14 & 32 & 150 & 4.01 \\
\hline & 62 & -24 & 12 & & 3.93 \\
\hline (C) Empathic concern & 60 & -14 & 22 & & 3.76 \\
\hline \multirow[t]{3}{*}{ Precentral gyrus } & 64 & 4 & 20 & 817 & 4.77 \\
\hline & 52 & -10 & 10 & & 4.43 \\
\hline & 56 & -8 & -4 & & 4.33 \\
\hline
\end{tabular}


Table 2 continued

\begin{tabular}{|c|c|c|c|c|c|}
\hline Region & $x$ & $y$ & $z$ & $k$ & $t$ \\
\hline \multirow[t]{3}{*}{ Posterior cingulate cortex } & 6 & -54 & 8 & \multirow[t]{3}{*}{1,390} & 4.46 \\
\hline & 18 & -48 & 32 & & 4.28 \\
\hline & -10 & -76 & 30 & & 4.26 \\
\hline \multirow[t]{3}{*}{ MPFC } & -16 & 52 & 12 & \multirow[t]{3}{*}{197} & 4.31 \\
\hline & -10 & 64 & 12 & & 4.03 \\
\hline & -24 & 68 & 14 & & 3.67 \\
\hline \multirow[t]{3}{*}{ Superior frontal gyrus } & 36 & 60 & 18 & \multirow[t]{3}{*}{167} & 4.09 \\
\hline & 34 & 56 & 26 & & 3.96 \\
\hline & 54 & 32 & 26 & & 3.88 \\
\hline
\end{tabular}

Brain regions showing increased activation during the friend's exclusion (vs. inclusion) activation as a function of (A) interdependence, (B) perspective-taking and (C) empathic concern

response to a friend's exclusion (vs. inclusion) but negatively correlated with MPFC in response to a stranger's exclusion (vs. inclusion). These subscales ask participants to report on their own ability and tendency to empathize, but do not distinguish between empathizing for close others and strangers. Our neural data may suggest that individuals with the most empathic abilities, as measured by self-reports, may also be the most sensitive to motivational biases in putting their empathic abilities to use. However, this possibility cannot be tested with the current data and thus future studies will be needed to replicate and fully understand this observation.

It is noteworthy that our self-reported and neural effects related to empathy were specific to the perspective-taking and empathic concern subscales of the Empathy IRI (and not the fantasy and personal distress subscales). In our view, perspectivetaking and empathic concern can be considered 'other-focused' components of empathy in that they emphasize the thoughts (perspective-taking) and feelings toward (empathic concern) another target. In contrast, the fantasy subscale, which measures the ability to identify with fictional characters, and personal distress subscale, which measures the degree of anxiety and discomfort felt in response to another person's distress, may be conceived as more 'self-focused' components of empathy in that they both allow the observer to experience the target's situation first-hand. Interestingly, this distinction fits with earlier suggestions that interdependent self-construal may specifically relate to other-focused emotions and social cognitive abilities (Markus and Kitayama 1991). Thus, an interesting future direction will be to examine how interdependence affects experimentally manipulated other-focused and self-focused psychological states, as well as the role of MPFC in any observed effects.

Our results also beg the question of whether there may be cultural differences in empathy. The purpose of the current study was to unravel the neural mechanisms linking interdependence to empathy, and so we focused on a participant population (Chinese nationals) with strong, but also variable levels of interdependence. However, it is well known that individuals from Eastern and Western cultures vary 
Table 3 Whole-brain regression results

Decreases during the stranger's exclusion (vs. inclusion)

\begin{tabular}{|c|c|c|c|c|c|c|}
\hline Region & Laterality & $x$ & $y$ & $z$ & $k$ & $t$ \\
\hline \multicolumn{7}{|l|}{ (A) Interdependence } \\
\hline \multirow[t]{3}{*}{ MPFC } & $\mathrm{L}$ & -12 & 60 & 16 & 275 & 5.19 \\
\hline & $\mathrm{L}$ & -12 & 54 & 22 & & 4.86 \\
\hline & $\mathrm{L}$ & -6 & 44 & 22 & & 4.7 \\
\hline \multirow[t]{3}{*}{ DMPFC } & $\mathrm{L}$ & -12 & 38 & 44 & 219 & 5.18 \\
\hline & $\mathrm{L}$ & -16 & 32 & 38 & & 5.13 \\
\hline & $\mathrm{L}$ & -22 & 40 & 48 & & 4.57 \\
\hline \multirow[t]{3}{*}{ Inferior parietal cortex } & $\mathrm{L}$ & -60 & -50 & 40 & 924 & 7.33 \\
\hline & $\mathrm{L}$ & -58 & -52 & 30 & & 6.75 \\
\hline & $\mathrm{L}$ & -48 & -56 & 36 & & 5.8 \\
\hline \multicolumn{7}{|l|}{ (B) Perspective-taking } \\
\hline \multirow[t]{3}{*}{ MPFC } & $\mathrm{L}$ & -16 & 68 & 8 & 614 & 6.95 \\
\hline & $\mathrm{R}$ & 4 & 64 & 18 & & 5.86 \\
\hline & $\mathrm{L}$ & -16 & 60 & 14 & & 5.35 \\
\hline \multirow[t]{3}{*}{ Anterior insula } & $\mathrm{L}$ & -26 & 20 & -8 & 277 & 6.07 \\
\hline & $\mathrm{L}$ & -32 & 32 & -12 & & 4.53 \\
\hline & $\mathrm{L}$ & -24 & 6 & -12 & & 4.31 \\
\hline \multirow[t]{2}{*}{ Postcentral gyrus } & $\mathrm{R}$ & 40 & -28 & 64 & 200 & 6.91 \\
\hline & $\mathrm{R}$ & 48 & -30 & 58 & & 5.28 \\
\hline \multirow[t]{3}{*}{ Postcentral gyrus } & $\mathrm{R}$ & 48 & -30 & 58 & 906 & 6.25 \\
\hline & $\mathrm{L}$ & -54 & -8 & -32 & & 5.66 \\
\hline & $\mathrm{L}$ & -44 & -30 & -24 & & 4.9 \\
\hline \multirow[t]{3}{*}{ Ventrolateral prefrontal cortex } & $\mathrm{L}$ & -32 & 64 & 0 & 503 & 5.63 \\
\hline & $\mathrm{L}$ & -38 & 46 & -20 & & 5.3 \\
\hline & $\mathrm{L}$ & -38 & 54 & -18 & & 5.07 \\
\hline \multirow[t]{3}{*}{ Fusiform gyrus } & $\mathrm{R}$ & 58 & -2 & -28 & 225 & 5.07 \\
\hline & $\mathrm{R}$ & 46 & 2 & -38 & & 4.96 \\
\hline & $\mathrm{R}$ & 46 & 0 & -50 & & 4.92 \\
\hline \multirow[t]{3}{*}{ Inferior frontal gyrus } & $\mathrm{L}$ & -46 & 24 & -4 & 320 & 4.82 \\
\hline & $\mathrm{L}$ & -54 & 12 & -18 & & 4.07 \\
\hline & $\mathrm{L}$ & -50 & 14 & -10 & & 3.78 \\
\hline \multicolumn{7}{|l|}{ (C) Empathic concern } \\
\hline \multirow[t]{3}{*}{ Middle temporal gyrus } & $\mathrm{R}$ & 52 & 0 & -34 & 157 & 4.37 \\
\hline & $\mathrm{R}$ & 44 & 4 & -38 & & 4.09 \\
\hline & $\mathrm{R}$ & 44 & -2 & -32 & & 3.94 \\
\hline
\end{tabular}

Brain regions showing decreased activation during the stranger's exclusion (vs. inclusion) as a function of (A) interdependence, (B) perspective-taking and (C) empathic-concern

in the extent to which they endorse interdependence and independence (or, perceiving oneself and one's experiences as unique from, or independent from, others; Markus and Kitayama 1991, 2010). Moreover, research on culture gene- 
coevolution suggests that Eastern cultures, relative to Western cultures, may show a greater prevalence of individuals carrying social sensitivity genes relevant to empathic processes (Chiao et al. 2012; Way and Lieberman 2010). Importantly, we do not hypothesize that endorsing independence, relative to interdependence, would be associated with less outgroup biases in empathy. On the contrary, we suspect that because both forms of construal sharpen the boundary between the self and others, both should be associated with reduced empathy to others separate from the self. Thus, relative to interdependence, independence may either be associated with less empathy in general, or specifically less empathy towards close others. Along these lines, in addition to general cross-cultural comparisons on the neural basis of empathy, future research should examine whether, across cultures, a basic principle of empathy is that the degree of empathic neural responding to a target follows a gradient of how conceptually tied the target is to the self.

More generally, our findings offer interesting insight into the role of MPFC in empathy. While a large body of research suggests that dACC and AI are basic mechanisms supporting vicarious emotional responses in empathy (e.g., Gu and Han 2007; Han et al. 2009; Jackson et al. 2005; Singer et al. 2004; Zaki and Ochsner 2012), other findings suggest that MPFC may also be pivotal to empathy. For example, MPFC activation is associated with empathy for negative social and emotional experiences (Bruneau et al. 2012b; Masten et al. 2011; Morelli et al. 2012; Zaki et al. 2009) and greater self-reported empathic behavior over the course of several days (Rameson et al. 2012). Interestingly, as in our study, all of the above findings surround empathy for social and emotional experiences, whereas the literature emphasizing the importance of $\mathrm{dACC}$ and $\mathrm{AI}$ activation focus on empathy for others' physical pain. One possibility is that MPFC plays a general role in representing social-conceptual information, which facilitates empathizing for suffering that is mental, rather than physical. Our findings fit with this interpretation, as MPFC's neural response appeared to track with the social saliency of the target. That is, degree of interdependence moderated MPFC activation for a friend's and stranger's exclusion, suggesting this region was sensitive to the social relationship between a perceiver and a target, and this sensitivity related to trait empathy.

\section{Limitations}

It is important to note that data from this sample has been previously published (Meyer et al. 2013). In Meyer et al. (2013), our goal was to examine how closeness with a target may close empathy gaps for social suffering. We found that, on average, empathy for a friend's social exclusion (vs. inclusion) corresponded with activation in and functional connectivity between MPFC, dACC, and AI. In contrast, empathy for a stranger's exclusion (vs. inclusion) was associated with neural activity in brain regions supporting thinking about other people's intentions (e.g., DMPFC). In the present study, we use neural data from independent ROIs that were not used in the previously published paper to help answer a different theoretical question: does trait interdependence correspond with a general increase in empathy, or does trait interdependence enhance empathy for close others, but decrease empathy for strangers? Because this question is theoretically distinct from 
the question examined in the previous publication, and because the statistical tests reported here are orthogonal (e.g., statistically independent) to those previously reported, the results reported here add important (albeit incremental) insight into how cultural influences of trait interdependence may influence empathy. Nonetheless, future research in new, larger samples of individuals from both interdependent and independent cultures will help further clarify how cultural influences on interdependence and independence affects empathy.

\section{Conclusion}

In conclusion, our study provides the first neuroimaging evidence in support of the idea that the mechanisms linking interdependence to empathy differentiate between close others and strangers. Specifically, we found that MPFC activation when observing a friend's exclusion (vs. inclusion) positively correlated with trait interdependence and empathy, whereas MPFC activation when observing a stranger's exclusion (vs. inclusion) negatively correlated with trait interdependence and empathy. Future research will help clarify whether this neural differentiation varies across cultures known to vary along the interdependence and independence continuums.

Acknowledgments This work was supported by the National Natural Science Foundation of China (Project 30910103901, 91024032, 81161120539; 91224008), the National Basic Research Program of China (973 Program 2010CB833903), Beijing Municipal Natural Science Foundation (No. Z111107067311058) and the U.S. National Science Foundation East Asia and Pacific Summer Institute Fellowship.

\section{References}

Avenanti, A., Sirigu, A., \& Aglioti, S. M. (2010). Racial bias reduces empathic sensorimotor resonance with other-race pain. Current Biology, 20(11), 10.

Bruneau, E. G., Dufour, N., \& Saxe, R. (2012a). Social cognition in members of conflict groups: Behavioural and neural responses in Arabs, Israelis and South Americans to each other's misfortunes. Philosophical Transactions of the Royal Society of London, Series B: Biological sciences, 367(1589), 717-730.

Bruneau, E. G., Pluta, A., \& Saxe, R. (2012b). Distinct roles of the 'shared pain' and 'theory of mind' networks in processing others' emotional suffering. Neuropsychologia, 50(2), 219-231.

Chiao, J. Y., Blizinsky, K. D., Mathur, V. A., \& Cheon, B. K. (2012). Culture-gene coevolution of empathy and altruism. In B. Oakley, A. Knafo, G. Madhavan, \& D. Wilson (Eds.), Pathological altruism. Oxford: Oxford University Press.

Chiao, J. Y., Harada, T., Komeda, H., Li, Z., Mano, Y., Saito, D., et al. (2009). Neural basis of individualistic and collectivistic views of self. Human Brain Mapping, 30(9), 2813-2820.

Chiao, J. Y., Harada, T., Komeda, H., Li, Z., Mano, Y., Saito, D., et al. (2010). Dynamic cultural influences on neural representations of the self. Journal of Cognitive Neuroscience, 22(1), 1-11.

Cox, R. W. (1996). AFNI: Software for analysis and visualization of functional magnetic resonance neuroimages. Computational Biology Research, 29, 162-173.

D’Argembeau, A., Ruby, P., Collette, F., Degueldre, C., Balteau, E., Luxen, A., et al. (2007). Distinct regions of the medial prefrontal cortex are associated with self-referential processing and perspective taking. Journal of Cognitive Neuroscience, 19(6), 935-944.

Danziger, N., Faillenot, I., \& Peyron, R. (2009). Can we share a pain we never felt? Neural correlates of empathy in patients with congenital insensitivity to pain. Neuron, 61(2), 203-212. 
Davis, M. (1983). Measuring individual differences in empathy: Evidence for a multidimensional approach. Journal of Personality and Social Psychology, 44(1), 113-126.

Denny, B. T., Kober, H., Wager, T. D., \& Ochsner, K. N. (2012). A meta-analysis of functional neuroimaging studies of self- and other judgments reveals a spatial gradient for mentalizing in medial prefrontal cortex. Journal of Cognitive Neuroscience, 24(8), 1742-1752.

Eisenberger, N. I., \& Lieberman, M. D. (2004). Why rejection hurts: A common neural alarm system for physical and social pain. Trends in Cognitive Science, 8(7), 294-300.

Eisenberger, N. I., Lieberman, M. D., \& Williams, K. D. (2003). Does rejection hurt? An FMRI study of social exclusion. Science, 302(5643), 290-292.

Gardner, W., Gabriel, S., \& Dean, K. (2004). The individual as "melting pot": The flexibility of bicultural self-construals. Cahiers de Psychologie Cognitive, 22, 181-201.

Gu, X., \& Han, S. (2007). Attention and reality constraints on the neural processes of empathy for pain. Neuroimage, 36, 256-267.

Han, S., Fan, Y., Xu, X., Qin, J., Wu, B., Wang, X., et al. (2009). Empathic neural responses to others' pain are modulated by emotional contexts. Human Brain Mapping, 30, 3227-3237.

Harris, L. T., \& Fiske, S. T. (2006). Dehumanizing the lowest of the low: Neuroimaging responses to extreme out-groups. Psychological Science, 17(10), 847-853.

Harris, L. T., \& Fiske, S. T. (2007). Social groups that elicit disgust are differentially processed in mPFC. Social Cognitive and Affective Neuroscience, 2(1), 45-51.

Hein, G., Silani, G., Preuschoff, K., Batson, C. D., \& Singer, T. (2010). Neural responses to ingroup and outgroup members' suffering predict individual differences in costly helping. Neuron, 68(1), 149-160.

Iyengar, S. S., Lepper, M. R., \& Ross, L. (1999). Independence from whom? Interdependence with whom? Cultural perspectives on ingroups versus outgroups. In D. A. Prentice \& D. T. Miller (Eds.), Cultural divides: Understanding and overcoming group conflict. New York: Russell Sage.

Jackson, P. L., Brunet, E., Meltzoff, A. N., \& Decety, J. (2006). Empathy examined through the neural mechanisms involved in imagining how I feel versus how you feel pain. Neuropsychologia, 44(5), 752-761.

Jackson, P. L., Meltzoff, A. N., \& Decety, J. (2005). How do we perceive the pain of others? A window into the neural processes involved in empathy. Neuroimage, 24(3), 771-779.

Jiang, C., Varunum, M., Hou, Y., \& Han, S. (2013). Distinct effects of self-construal priming on empathic neural responses in Chinese and Westerners. Social Neuroscience, 9(2), 130-138.

Kelley, W. M., Macrae, C. N., Wyland, C. L., Caglar, S., Inati, S., \& Heatherton, T. F. (2002). Finding the self? An event-related fMRI study. Journal of Cognitive Neuroscience, 14(5), 785-794.

Lamm, C., Batson, C. D., \& Decety, J. (2007). The neural substrate of human empathy: Effects of perspective-taking and cognitive appraisal. Journal of Cognitive Neuroscience, 19(1), 42-58.

Leung, K. (1988). Some determinants of conflict avoidance. Journal of Cross-Cultural Psychology, 19, $125-136$.

Leung, K., \& Bond, M. H. (1984). The impact of cultural collectivism on reward allocation. Journal of Personality and Social Psychology, 47, 793-804.

Ma, Y., Bang, D., Wang, C., Allen, M., Frith, C., Roepstorff, A., \& Han, S. (2014). Sociocultural patterning of neural activity during self-reflection. Social Cognitive and Affective Neuroscience, 9, 73-80.

Ma-Kellams, C., \& Blascovich, J. (2012). Inferring the emotions of friends versus strangers: The role of culture and self-construal. Personality and Social Psychology Bulletin, 38, 933-945.

Maldjian, J. A., Laurienti, P. J., Kraft, R. A., \& Burdette, J. H. (2003). An automated method for neuroanatomic and cytoarchitectonic atlas-based interrogation of fMRI data sets. Neuroimage, 19(3), 1233-1239.

Markus, H., \& Kitayama, S. (1991). Culture and self: Implications for cognition, emotion, and motivation. Psychological Review, 98, 224-253.

Markus, H. R., \& Kitayama, S. (2010). Cultures and selves: A cycle of mutual constitution. Perspectives on Psychological Science, 5(4), 420-430.

Masten, C. L., Morelli, S. A., \& Eisenberger, N. I. (2011). An fMRI investigation of empathy for 'social pain' and subsequent prosocial behavior. Neuroimage, 55(1), 381-388.

Meyer, M. L., Masten, C. L., Ma, Y., Wang, C., Shi, Z., Eisenberger, N. I., et al. (2013). Empathy for the social suffering of friends and strangers recruits distinct patterns of brain activation. Social Cognitive and Affective Neuroscience, 8(4), 446-454. 
Morelli, S. A., Rameson, L. T., \& Lieberman, M. D. (2012). The neural components of empathy: Predicting daily prosocial behavior. Social Cognitive and Affective Neuroscience.

Ng, S. H., Han, S., Mao, L., \& Lai, J. C. L. (2010). Dynamic bicultural brains: A fMRI study of their flexible neural representation of self and significant others in response to culture priming. Asian Journal of Social Psychology, 13, 83-91.

Rameson, L. T., Morelli, S. A., \& Lieberman, M. D. (2012). The neural correlates of empathy: Experience, automaticity, and prosocial behavior. Journal of Cognitive Neuroscience, 24(1), 235-245.

Sheng, F., \& Han, S. (2012). Manipulations of cognitive strategies and intergroup relationships reduce the racial bias in empathic neural responses. Neuroimage, 61, 786-797.

Sheng, F., Liu, Y., Zhou, B., Zhou, W., \& Han, S. (2013). Oxytocin modulates the racial bias in neural responses to others' suffering. Biological Psychology, 92, 380-386.

Singer, T., Seymour, B., O’Doherty, J., Kaube, H., Dolan, R. J., \& Frith, C. D. (2004). Empathy for pain involves the affective but not sensory components of pain. Science, 303(5661), 1157-1162.

Smith, S. M. (2002). Fast robust automated brain extraction. Human Brain Mapping, 17, 143-155.

Steiger, J. H. (1980). Tests for comparing elements of a correlation matrix. Psychological Bulletin, 87, 245-251.

Triandis, H. C. (1972). The analysis of subjective culture. New York: Wiley.

Triandis, H. C. (1989). The self and social behavior in differing cultural contexts. Psychological Review, 96(3), 506-520.

Triandis, H. C., \& Gelfand, M. J. (1998). Converging measurement of horizontal and vertical individualism and collectivism. Journal of Personality and Social Psychology, 74(1), 118-128.

Tzourio-Mazoyer, N., Landeau, B., Papathanassiou, D., Crivello, F., Etard, O., Delcroix, N., et al. (2002). Automated anatomical labeling of activations in SPM using a macroscopic anatomical parcellation of the MNI MRI single-subject brain. Neuroimage, 15(1), 273-289.

Wang, C., Oyserman, D., Li, H., Liu, Q., \& Han, S. (2013). Accessible cultural mindset modulates default mode activity: Evidence for the culturally situated brain. Social Neuroscience, 8, 203-216.

Way, B. M., \& Lieberman, M. D. (2010). Is there a genetic contribution to cultural differences? Collectivism, individualism and genetic markers of social sensitivity. Social Cognitive and Affective Neuroscience, 5(2-3), 203-211.

Williams, E. J. (1959). The comparison of regression variables. Journal of the Royal Statistical Society (Series B), 21, 396-399.

Woltin, K.-A., Yzerbyt, V. Y., \& Corneille, O. (2011). On reducing an empathy gap: The impact of selfconstrual and order of judgment. British Journal of Social Psychology, 50, 553-562.

Wong, R. Y.-M., \& Hong, Y.-Y. (2005). Dynamic influences of culture on cooperation in the prisoner's dilemma. Psychological Science, 16, 429-434.

Wu, S., \& Keysar, B. (2007). The effect of culture on perspective-taking. Psychological Science, 18, 600-606.

Xu, X., Zuo, X., Wang, X., \& Han, S. (2009). Do you feel my pain? Racial group membership modulates empathic neural responses. Journal of Neuroscience, 29(26), 8525-8529.

Zaki, J. (2014). Empathy: A motivated account. Psychological Bulletin, 140(6), 1608-1647.

Zaki, J., Bolger, N., \& Ochsner, K. (2009a). Unpacking the informational bases of empathic accuracy. Emotion, 9(4), 478-487.

Zaki, J., \& Ochsner, K. (2012). The neuroscience of empathy: Progress, pitfalls and promise. Nature Neuroscience, 15(5), 675-680.

Zaki, J., Weber, J., Bolger, N., \& Ochsner, K. (2009b). The neural bases of empathic accuracy. Proceedings of the National Academy of Sciences USA, 106(27), 11382-11387.

Zhu, Y., Zhang, L., Fan, J., \& Han, S. (2007). Neural basis of cultural influence on self-representation. Neuroimage, 34(3), 1310-1316. 